Physical activity levels and barriers to exercise in people living with a stoma

Bethany Lowe, RGN, MSc [corresponding author]

Deputy Ward Sister,

Nottingham University Hospitals NHS Trust, Nottingham, UK.

Dr Eman Alsaleh, RGN, PhD

Lecturer in Nursing,

Philadelphia University, Amman, Jordan.

Dr Holly Blake ${ }^{1,2}$, BA(Hons), PhD, CPychol

Associate Professor of Behavioural Science,

${ }^{1}$ University of Nottingham, UK.

${ }^{2}$ NIHR Biomedical Research Centre, Nottingham, UK.

\title{
Acknowledgements
}

The authors would like to thanks Natalia Stanulewicz for supporting the statistical analysis in this study.

\section{Conflicts of Interests}

No conflict of interest has been declared by the authors.

\section{Funding}

This research received no specific grant from any funding agency in the public, commercial, or notfor-profit sectors. 


\section{Abstract}

Background: Physical activity is important for physical and mental health although people with a stoma commonly experience a reduction in physical activity following their stoma formation.

Aim: To assess whether adults with a stoma achieve the recommended amount of physical activity, and to identify any barriers and facilitators that may influence their participation.

Method: Community-residing adults living with a urostomy, ileostomy and colostomy were recruited from six regional stoma support groups. Participants completed questionnaires assessing their physical activity levels, self-efficacy, perceived barriers and benefits to physical activity, depression, body image and stoma-related quality of life.

Results: 94 adults completed the questionnaire ( $81 \%$ response rate). $84 \%$ of participants did not achieve government recommended levels of physical activity. Less active participants perceived greater barriers to physical activity, and had lower self-efficacy for exercise than those who were more active. Reported physical activity was not associated with body image, depression or stomarelated quality of life.

Conclusion: Most of the community-residing adults living with a stoma in our sample were physically inactive. Interventions that reduce barriers to exercise, and build self-efficacy in people with a stoma may help to increase their physical activity levels and reduce risks of chronic disease associated with sedentary lifestyles. 


\section{Background}

It is estimated that around one in $\mathbf{5 0 0}$ people in the UK are living with a stoma (Boyles and Hunt, 2016). A number of conditions may require stoma formation, including colorectal cancer, diverticular disease, ulcerative colitis and Crohn's disease (Taylor, 2005). The three types of eliminating stomas are colostomy, ileostomy and urostomy, and they can be temporary or permanent (Hubbard et al, 2017). Having a stoma can impact on quality of life (Russell, 2017; Hubbard et al, 2017). A recent systematic review identified reports of sexual problems, depressive feelings, gas, constipation, dissatisfaction with appearance, change in clothing, travel difficulties and tiredness (Vonk-Klassenn, et al, 2016). People with a stoma report feeling less confident to undertake activities they engaged with before the stoma formation ( $\mathrm{Wu}$ et al, 2007) and may experience altered body image, low self-confidence and low self-efficacy (Ross et al, 2007; Dabirian et al, 2010). Self- efficacy is an individuals' belief in their ability to complete tasks and succeed (Bandura, 1977), and is strongly associated with adjustment to stoma care (Simmons et al, 2007) and the uptake and maintenance of healthy lifestyle behaviours, such as physical activity (Lachman et al, 2018).

Being physically active is important for general health with regards the prevention of noncommunicable disease, reducing rates of mortality, increasing independence and overall life expectancy (WHO, 2018). In 2015/16, 26\% of UK adults were classified as inactive (engaging in fewer than 30 minutes physical activity a week) (NHS Digital, 2017). Rates of physical activity participation in people living with a stoma are not well established, although a national survey identified a trend towards physical inactivity after stoma surgery and a fear of exercise (Russell, 2017). People living with long-term conditions experience greater barriers to physical activity and may be less active than the general population (WHO, 2018; Blake, 2010). People with a stoma report low adjustment to participating in sports and physical activities (Fingren et al, 2018) and a reduction in physical activities following their stoma formation (Dabirian et al, 2010).

The level of physical activity individuals engage in is often dependent on the nature of their condition, individual needs, beliefs about their ability and fear of worsening their condition. In those for whom physical activity is not contraindicated, efforts should be made to empower individuals with the skills and confidence to engage in active lifestyles. To make appropriate recommendations for clinical support, there is a need for research to further investigate physical activity levels in people living with a stoma, and to determine which factors are associated with engagement in regular physical activity, or conversely with inactivity.

\section{Aims}

The primary aim of this study was to assess reported physical activity levels in community-dwelling adults living with a stoma. The secondary aim was to investigate the relationship between physical

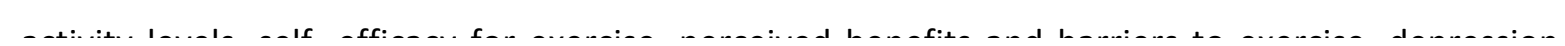


body image, and stoma-related QoL.

\section{Methods}

\section{Study Design}

This study was a cross-sectional questionnaire survey undertaken with a convenience sample of healthy individuals living with a stoma, recruited via six regional stoma support groups.

\section{Measures}

The primary measure was physical activity. Secondary measures included self-efficacy for exercise, benefits and barriers to exercise, depression, body image and stoma-related QoL.

\section{Physical Activity}

The International Physical Activity Questionnaire short-form (IPAQ, 2004) was used to assess participants' self- reported physical activity levels. Participants are asked to report on the frequency (number of days per week) and duration (hours and minutes per day) spent walking, engaging in moderate intensity activities and vigorous intensity activities. Scores are calculated using the metabolic equivalent of task (MET) value. Each activity is weighted against the energy requirements of the activity to produce a score in MET-minutes. Scores determine whether the individual is 'inactive', 'minimally active' or 'HEPA active' (health-enhancing physical activity). Participant METs are shown in Table 1.

\section{Self-Efficacy for Exercise}

The Self-Efficacy for Exercise Scale (SEES) was used to assess participants' confidence to exercise (Resnick and Jenkins, 2000). This scale outlines nine situations that can affect participation in physical activity and focuses on an individual's confidence in their ability to engage in exercise despite these barriers. Participants are asked to rank their confidence to 'exercise three times per week for 20 minutes', on a scale of $0-10$, where $0=$ not confident and $10=$ very confident. An overall score is calculated for each participant. Possible scores range from 0-90. Higher scores indicate a greater level of self-efficacy for exercise.

\section{Benefits and Barriers to Exercise}

Participants' perceptions of the benefits and barriers to physical activity were assessed using the Exercise Benefits and Barriers Scale - Adult Version (EBBS) (Sechrist et al, 1987). This scale consists of two component measures: Benefits and Barriers. The Benefits measure includes 29 items categorised into: life enhancement, physical performance, psychological outlook, social interaction and preventative health. The Barriers measure includes 14 items categorised into: exercise milieu, time expenditure, physical exertion, and family discouragement. Participants are asked to what 
likert-type scale, where 1= strongly disagree, 2= disagree, 3=agree, 4= strongly agree. Scores for each subscale are calculated, and the barriers subscale is reverse scored. Possible total scale scores range from 43-172. Higher total scores indicate more perceived benefits of physical activity.

\section{Depression}

The Patient Health Questionnaire PHQ-9 (Kroenke et al, 2001) is a self-report version of the PRIMEMD diagnostic instrument of mental disorders. This measure focuses on depression and is based on the DSM-V1 criteria. Participants are asked how often they have been bothered by mood states over the past two weeks. A likert-type scale is used: $0=$ not at all, $1=$ several days, $2=$ more than half days and $3=$ nearly every day. The total score for each participant is calculated to determine the PHQ-9 severity score. The maximum score is 27 (indicating no sign of depression). Scores of 5, 10, 15 and 20 represent borders for mild, moderate, moderately severe and severe depression, respectively.

\section{Body Image}

The Social Physique Anxiety Scale (Hart et al, 1989) has been used to measure the anxiety that an individual feels when it is perceived that others may be evaluating their physique. This 12 -item scale asks participants to rank how characteristic each statement is on a 5-point likert-type scale, where $1=$ not at all characteristic of me, $2=$ =slightly characteristic of me, $3=$ moderately characteristic of me, $4=$ very characteristic of me, and $5=$ extremely characteristic of me. The total score is combined for each participant, with a maximum score of

60. Lower scores indicate less physique anxiety.

\section{Stoma-Related Quality of Life}

Quality of life (QoL) was measured by the Stoma-Related QoL Questionnaire (Prieto et al, 2001). This includes 20 items covering five domains: stoma appliance, sleep, sexual activity, relationships with family/friends and social relationships. Participants are asked to answer the questions based on how they are feeling at the present time, on a 4-point likert-type scale: 1=always, 2=sometimes, $3=$ rarely and $4=$ not at all. The combined score for each participant is calculated. The highest possible score for the questionnaire is 60 (best QOL) and the lowest score is 0 (worst QOL).

\section{Procedure}

Ethical approval for this study was obtained from the local institutional review board for healthy volunteers (Ref: N12032015). Permission was granted from the leads of six regional support groups. Individual group members were eligible to take part if they had a stoma, attended a stoma 
group leaders to a total of 116 individuals attending one of the six groups and meeting the eligibility criteria. The researcher attended one meeting for each group within the region and gave a brief presentation on the nature and purpose of the research. It was communicated that participation was voluntary, all responses would be treated in confidence and accessible only to the research team, and no personal identifying information was being requested. Ethical issues relating to confidentiality, withdrawal and data protection were discussed with the group attendees. Assumed consent was taken by receipt of the completed questionnaire. Participants could choose to return the completed questionnaire in the mail, or leave it in with the group lead in a drop-box for collection by the researcher.

\section{Data analysis}

Data were analysed using SPSS (IBM PASW Version 22.0). Descriptive analysis was undertaken. Mean values between populations and MET Intensity levels were analysed using a one-way ANOVA test. A F-statistic (F) is stated within the results and a $p$-value of $<0.05$ was used to interpret significance.

\section{Results}

Data were collected during April to August 2015. Of the 116 individuals registered at the groups, $100 \%$ met the eligibility criteria. Of these, $94(81 \%)$ chose to complete the questionnaire.

\section{Sample Characteristics}

There were 48 women (51.1\%) and 46 men (48.9\%). Age ranged from 20 - 90 years $(n=94$, mean $=53.5$ years, SD=21.3 years). Ethnic origin included White British/Irish ( $n=70,74.5 \%)$, Mixed White/Black Caribbean ( $n=6,6.4 \%)$, Mixed White/Black African ( $n=3,3.2 \%)$, Mixed White/Asian $(n=3,3.2 \%)$, Asian Indian ( $n=10,10.6 \%)$, Asian Other $(n=1,1.1 \%)$, and Black African $(n=1,1.1 \%)$. Of respondents, 54 (57.4\%) were married or living with a partner, 18 (19.1\%) were single or never married, 21 were widowed, divorced or separated (22.3\%) and one preferred not to say (1.1\%). Over three-quarters of respondents reported having further or higher education qualifications or training beyond high school $(n=73,77.6 \%)$. Approximately one-third $(n=35,37.3 \%)$ of respondents were working (employed $n=31,33 \%$; self-employed $n=4,4.3 \%$ ).

Seventy-four respondents reported their stoma type, which included colostomy ( $N=30,31.9 \%)$, ileostomy ( $N=39,41.5 \%)$ or urostomy $(N=5,5.3 \%)$. Twenty participants did not respond or reported that they did not know their stoma type. Two thirds of participants had a permanent stoma $(\mathrm{N}=63$, $67 \%)$, one third had a temporary stoma ( $N=29,30.9 \%)$, and two participants did not respond. Figure 1 identifies physical activity levels (MET intensity as described below) for each stoma type. 
months). Data from participants who did not answer the stoma demographic questions were not included in the data analysis. Sample characteristics are provided in Table 1.

[insert Figure 1 here]

\section{International Physical Activity Questionnaire}

MET Intensity values were calculated for each participant and categorised into three groups: inactive, minimally active and HEPA active. Three-quarters of participants were either inactive or minimally active ( $n=71,75.5 \%)$. Participants were categorised as inactive $(n=36,38.3 \%)$, minimally active ( $n=35,37.2 \%)$ and HEPA active $(n=15,16 \%)$, respectively (see Table 1$)$. There was a significant gender difference between categories with HEPA active participants marginally more likely to be female than male $(p=0.05)$.

\section{[Insert Table 1 here]}

\section{Self-Efficacy for Exercise Scale}

Mean SES score (mean 40.8, SD 20.7) indicated moderate self-efficacy. There was a significant effect for SES scores on MET Intensity levels $(F=3.04, p<0.001)$. The SES mean score for inactive groups ( $n=34, M=30.4$ ) was significantly lower than SES scores for both minimally active groups ( $n=33, M=46, p=0.03)$ and HEPA active groups $(n=15, M=49, p=0.01$ ). There was no statistically significant difference in SES scores between minimally active groups and HEPA active groups $(p=0.61)$. Mean scores for each of the SES questions were calculated to determine the factors that are perceived to be the greatest challenge to confidence about participating in exercise. Scores were ranked from lowest to highest. Participants had the lowest levels of self-efficacy when they felt pain when exercising. Results are shown in Table 2.

[Insert Table 2 here]

\section{Patient Health Questionnaire}

The total score for each participant was calculated to determine the severity of depression score in line with the PHQ-9 scoring protocol (PHQ, 2002). Participants who did not fully complete the PHQ9 were not included in data analysis $(\mathrm{N}=4,4.3 \%)$. Mean depression severity score was 3.22 $(S D=4.8)$. When categorised, $66(70.2 \%)$ respondents showed no signs of depression, and 34 (29.8\%) showed signs of depression (mild: $n=14,15 \%$; moderate: $n=6,6.4 \%$; moderately severe: 
scores and lower levels of physical activity as assessed by the IPAQ although this did not reach statistical significance. Those participants showing signs of depression were more likely to be MET intensity classified as physically inactive $(n=19,58 \%)$ than minimally $(n=11,33 \%)$ or HEPA active $(n=3,9 \%)$. Results are shown in Table 3.

[insert Table 3 here]

\section{Exercise Benefits and Barriers Scale (EBBS)}

Barriers and benefits were ranked from lowest to highest. The factors perceived to be the greatest barriers to physical activity were associated with physical exertion, time and accessibility. The factors perceived as benefits to physical activity were primarily focused on the benefits to health. Scores for the total EBBS questionnaire ranged from 43-172 (mean=74, SD=15.8). A One-Way ANOVA test was conducted to compare the total EBBS score to MET Intensity levels. There was a significant effect for total EBBS score and MET intensity levels $(F=8.2, p=0.01)$. EBBS scores were significantly lower in physically inactive participants $(n=33, M=66, S D=12.2)$ compared to both minimally active participants $(n=33, M=79, S D=15.7, p=0.02)$ and HEPA active participants $(n=15$, $M=81, S D=18.4, p=0.05)$. Those with higher total EBBS score (with greater perceived benefits) were more likely to be HEPA or minimally active than inactive. Conversely, those with lower EBBS score (with fewer perceived benefits) were more likely to be physically inactive than minimally or HEPA active. No statistical significance was found between minimally active groups and HEPA active groups $(p=0.90)$.

To determine whether the perceived benefits and barriers were independently related to physical activity levels, analysis was conducted on separate scale scores. The mean benefit score was 53.5 $(S D=12.7)$ and the mean barrier score was $22.7(S D=6.6)$. Due to the barrier scale being reversescored, a higher barrier score indicates fewer barriers to physical activity. There were fewer barriers reported by participants with each increase in IPAQ classification category: inactive $(m=34$, $M=20.88, S D=6.08)$, minimally active $(n=34, M=23.08, S D=1.21)$, HEPA active $(n=15, M=26.6, S D=$ 1.7), although this did not reach statistical significance $(F=3.97, p=0.24)$. However, there was a significant effect for benefit scores on MET Intensity Levels ( $F=6.9, p=0.02)$. The means suggest that physically inactive participants perceived fewer benefits to exercise than minimally active or HEPA active participants. A Tukey HSD test indicated that mean benefit scores for inactive participants $(n=33, M=47, S D=1.7)$ were significantly lower compared to minimally active participants $(n=33$, $M=58, S D=13.3, p=0.01$ ). No significant difference was found when comparing HEPA active groups $(N=17, M=55, S D=14.2)$ to minimally active groups $(p=0.73)$ or inactive groups $(p=0.71)$. Results are shown in table 4. 


\section{Social Physique Anxiety Scale}

SPAS scores ranged from 12 to 52 (mean=30.83, SD=7.9), indicating moderate physical anxiety. There were no significance differences in SPAS scores between participants who were classified as inactive, minimally active or HEPA active, suggesting that within this sample, body confidence was unrelated to physical activity levels $(F=1.97, p=0.15)$. Mean scores for each of the SPAS items were calculated to determine factors that were associated with body confidence. Question items were then ranked in order of lowest to highest. The top five ranked questions were all associated with negative perceptions of body image and confidence. Results are shown in Table 4.

\section{Stoma-Related Quality of Life Questionnaire}

QoL scores ranged from 0-41 (mean=16.81, SD=9.7) indicating low $Q$ oL for the sample. Items were ranked by means to identify the top five factors perceived to have the greatest impact on QoL. "I become anxious when the (stoma) pouch is full" was the most commonly identified impact on QoL for these participants. A One-Way ANOVA test was used to compare perceived QoL to MET Intensity levels. No significant difference was found in QoL scores between MET intensity groups $(F=0.40, p=0.67)$ : Inactive $(n=35, M=18.17, S D=9.85)$, minimally active $(n=33, M=16.03, S D=10.30)$, and HEPA active $(n=17, M=16.82, S D=9.22)$. Results are shown in Table 4. [insert Table 4 here]

\section{Discussion}

Physical inactivity was prevalent in this sample, with $84 \%$ of our participants failing to achieve recommended physical activity levels. This is consistent with prior research showing reductions in physical activity following stoma formation (Dabirian et al, 2010; Russell, 2017). Although there is limited evidence around physical activity with stoma, this study suggests that they may be less physically active than the general population. The rates of physical inactivity shown here are alarming since we used a self-report scale, and there is a tendency to over- rather than under-report physical activity using the IPAQ (Lee et al, 2011).

Although depression is common in adults with a stoma, our support-group sample did not report particularly low mood, although there was a non-significant tendency for those with lower mood to be less active. Women in this sample were more active than men, the reverse of patterns found in general population (Azevedo et al, 2007).

Self-efficacy was significantly lower in those who were classified as inactive, compared to those who engaged in either minimal or HEPA activity levels. The fact that no difference in self-efficacy was found between the two levels of 'activeness' suggests that the most important difference in self-efficacy lies between those who are completely sedentary and those who engage in some level of physical activity, even if this is minimal. It may be beneficial to focus on shifting those individuals who are completely inactive towards engagement in some level of physical activity.

The relationship between self-efficacy and physical activity participation is consistent with previous 
their self-efficacy level. It may be that healthcare professionals need to provide more specific guidance around being physically active with a stoma. This is important given recent evidence suggesting that patients do not recall being given any advice about exercise or physical activity (Russell, 2017). Health promotion efforts could focus on the positive benefits of exercise while helping to address barriers to being active. Pain when exercising, feelings of depression and tiredness were associated with lower levels of self-efficacy for exercise in this sample. Building confidence in people with a stoma to engage in more physical activity may help to alleviate low mood and may reduce tiredness, because active lifestyles can generate greater energy levels. To build self-efficacy for exercise, it may be necessary to help people with a stoma manage pain expectations around physical activity to help them to set achievable physical activity goals. Variations in condition severity, co-morbidities, self-perceptions and anxieties around worsening of the condition can influence self-efficacy and physical activity levels and should be taken into account when designing physical activity interventions.

Lack of time is the most commonly cited barrier to physical activity in the general population (Bautista et al, 2011). Whilst this was identified as a barrier in this sample, it was not the most prevalent issue. More commonly reported barriers to exercise were physical exertion and fatigue. Previous studies have similarly identified physical exertion as a barrier to physical activity in individuals with long-term conditions (Lynch et al, 2007).

Concerns about damaging the stoma, developing parastomal hernias and pain have been previously identified as barriers to physical activity in adults with a stoma (Wu et al, 2007). These condition-specific factors are not assessed by the EBBS. However, participants' responses on the SES did indicate that pain during exercise was one of the factors that would impact on exercise selfefficacy. Further investigation is needed around the management of concerns and expectations, which would inform approaches to increasing physical activity in this population.

Participants in this study were most likely to report perceived benefits of exercise that were related to their physical health (e.g. prevention of heart attacks and increasing fitness). Early research on a non-stoma sample has shown that improvement to health and fitness can be a significant motivation to engage in physical activity (Zunft et al, 1999). Improved psychological health, stress management, and self-esteem are known benefits of physical activity. However, psychological benefits were not commonly flagged in the EBBS by our sample, and this study showed no statistically significant relationship between depression and physical activity, or body image and physical activity. Although, it should be recognised that the majority of our sample did not report low mood or low self-efficacy, and so were reasonably healthy with regards psychological status.

People with a stoma often experience low self-confidence due to altered body image (Ross et al, 2007). Physique anxiety was moderate in this sample. The measure of body image highlighted that any reported physique anxiety was most often related to: body proportion, apprehension about 
image and levels of physical activity. It should be noted that there was a lower response to the body image measure within the questionnaire compared to the other measures. It may be that participants with more difficulties in this area chose not to complete it. Alternatively, it may be that physical activity participation is associated more closely with physical health factors rather than psychological factors, for people with a stoma.

QoL can vary between individuals with a stoma; for some QoL will be improved and for some QoL will be impaired (Dabirian et al, 2010). This was reflected in comments made by participants in this study. One participant referred to the stress of the stoma 'leaking without warning' and another reported that the stoma was the 'best thing to happen' to them with regards to improved QoL. QoL has shown to improve as individuals adapt to living with their stoma (Dabirian et al, 2010). Although general QoL has shown to enhance health and physical activity participation (Gill et al, 2013), stoma-related QoL was not significantly associated with physical activity levels in our sample. However, it has been proposed that attending a support group improves QoL, which may reflect a bias in the sample. Factors negatively impacting on QoL in this sample included anxiety when the pouch is full, and feeling sexually unattractive. These factors concur with previous research (Wu et al, 2007; Ross et al, 2007) and may be important considerations in the design of interventions to build self-efficacy for exercise, reduce anxiety and ultimately increase physical activity in people with a stoma. The timing of intervention may be important, since factors that affect QoL should be identified and addressed soon after stoma formation (Dabirian et al, 2010).

\section{Limitations}

The sample is limited to adults attending a support group and may not be representative of the entire stoma population. Those accessing support groups may feel a greater need for support and be less well adjusted to the stoma ( $\mathrm{Hu}$ et al, 2014). Conversely, as individuals who are actively seeking support they could be better adjusted, with better psychological wellbeing, social support and QoL compared with individuals not accessing support groups (Docherty, 2004). We used selfreport measures of physical activity and objective activity data was not collected. The study was cross-sectional and does not allow for assessment of changes in physical activity behaviour over time. A longitudinal study may demonstrate whether physical activity levels change as individuals adapt to living with a stoma. We did not collect data on other health conditions, healthcare service utilisation, or extent of professional support although there is potential for these factors to influence participants' health behaviours and wellbeing.

\section{Conclusion}

The majority of community-living adults with a stoma in our sample were inactive and did not achieve government recommended levels of physical activity. There is a need to increase physical 
lifestyles. Psychological factors were not associated with physical activity in this sample of adults attending support groups. Efforts should be made by healthcare professionals to address stomarelated barriers to exercise and provide education and support around physical activity with a stoma. Intervention should promote the health benefits of active lifestyles and foster self-efficacy for exercise, which is important for initiating and sustaining changes in health behaviour. Tailored physical activity advice and goals could be offered by nurses, according to individual patient condition and capability. Advice may include setting goals related to gentle walking with gradual increments in walking distance, provision of advice and support for engaging in specific activities (e.g. use of a stoma cap or a mini- pouch for swimming) and potential avoidance of physical activities that avoid rough physical contact. People with a stoma should be supported to manage their physical activity expectations, and to set appropriate physical activity goals that take into account stoma-related symptoms (including pain, and fear of pain). Strategies to build self-efficacy for exercise will be important for long-term maintenance of lifestyle changes. Positive attitudes towards the benefits of physical activity and greater self-efficacy will be evident even in those who are minimally active, and so there will be value even in shifting those who are completely sedentary to minimally active.

\section{Implications for Practice}

- Efforts should be made to address stoma-related barriers to exercise and provide education and support around physical activity with a stoma

- Healthcare professionals should promote the health benefits of active lifestyles, and foster self- efficacy for exercise, which is important for initiating and sustaining changes in health behaviour.

\section{References}

Azevedo, R, Araújo, P, Reichert, F, Siqueira, V, Da Silva, C, Hallal, C (2007) Gender differences in leisure-time physical activity. International Journal of Public Health, 52(1),8-15. doi:10.1007/s00038-006-5062-1.

Bandura, A (1977) Social learning theory. New York: General Learning Press.

Blake, H, 2010. Physical activity and long-term conditions. In: Blake, H, ed., Physical activity in rehabilitation and recovery NovaScience, New York, USA. pp 17-50 
Boyles A, Hunt S. Care and management of a stoma: maintaining peristomal skin health. (2016). British Journal of Nursing, 25 (7). https://doi.org/10.12968/bjon.2016.25.17.S14

Burch, J. (2008). Stoma Care. West Sussex : Wiley and Sons Ltd

Dabirian A, Yaghmaei F, Rassouli M, Tafreshi MZ. (2010). Quality of life in ostomy patients: a qualitative study. Patient Prefer Adherence. 5:1-5.

Docherty, A (2004) Experience, functions and benefits of a cancer support group, 55(1), 87-93.

Fingren J, Lindholm E, Petersén C, Hallén AM, Carlsson E. (2018). A Prospective, Explorative Study to Assess Adjustment 1 Year After Ostomy Surgery Among Swedish Patients. Ostomy Wound Manage. 64 (6): $12-22$.

Gill D, Hammond CC, Reifsteck EJ, Jehu CM, Williams RA, Adams MM, Lange EH, Becofsky K, Rodriguez E, Shang YT. (2013). Physical Activity and Quality of Life. J Prev Med Public Health. 46(Suppl 1): S28-S34.

Hart, Leary, Rejeski. (1989). Social Physique Anxiety Scale. Retrieved from: http://www.midss.org/sites/default/files/social_phys_anx.pdf.

Hu A, Pan Y, Zhang M, Zhang J, Zheng M, Huang M, Ye X, Wu X. (2014) Factors influencing adjustment to a colostomy in Chinese patients: a cross-sectional study. J Wound Ostomy Continence Nurs. 41(5): 455-9. doi: 10.1097/WON.0000000000000053.

Hubbard G, Taylor C, Beeken B, Campbell A, Gracey J, Grimmett C, Fisher A, Ozakinci G, Slater S, Gorely T. (2017). Research priorities about stoma-related quality of life from the perspective of people with a stoma: A pilot survey. Health Expect., 20 (6): 1421-1427. Published online 2017 Jul 4. doi: 10.1111/hex.12585 International Physical Activity Questionnaire (2004). Guidelines for Data Processing and Analysis of the International Physical Activity Questionnaire (IPAQ) - Short Form. Retrieved from: http://www.institutferran.org/documentos/scoring_short_ipaq_april04.pdf.

Kroenke, K, Spitzer, L, Williams, B. (2001). The PHQ-9: validity of a brief depression severity measure. Journal General Internal Medicine, 16(9), 606-13. 
Exercise: Behavioral Strategies to Increase Physical Activity in Sedentary Middle-Aged and Older Adults. Innov Aging. 2 (1):igy007. doi: 10.1093/geroni/igy007. Epub 2018 Apr 5.

Lee, H, Macfarlane, J, Lam, H, Stewart,M (2011). Validity of the International PA Questionnaire Short Form (IPAQ-SF): a systematic review. Int J Behav Nutr Phys Act, 21(8), 115. doi:

10.1186/1479-5868-8-115.

Lynch, B, Cerin, E, Owen, N, Atiken, J. (2007). Associations of leisure-time physical activity with quality of life in a large, population-based sample of colorectal cancer survivors. Cancer causes and control, 18 (7), 735-742.

NHS Digital (2017). Statistics on Obesity, Physical Activity and Diet, England. Published 30th March, 2017. Available at:

https://assets.publishing.service.gov.uk/government/uploads/system/uploads/attachment_data/fil e/613532/o bes-phys-acti-diet-eng-2017-rep.pdf

PHQ. (2002). Instruction Manual; Instructions for Patient Health Questionnaire (PHQ) and GAD-7 Measure. Retrieved from http://www.phqscreeners.com/instructions/instructions.pdf.

Public Health England (2013). Social and economic inequalities in diet and physical activity. Retrieved from

http://www.noo.org.uk/uploads/doc/vid_19253_Social_and_economic_inequalities_in_diet_and_p hysical_acti vity_04.11.13.pdf. (Accessed 15/5/2017)

Prieto L, Thorsen H, Juul K. (2005). Development and validation of a quality of life questionnaire for patients with colostomy or ileostomy. Health Qual Life Outcomes. 3: 62.

Resnick B, Jenkins LS. (2000). Testing the reliability and validity of the Self-Efficacy for Exercise scale. Nurs Res. 49(3):154-9.

Ross, L, Abild-Nielsen, A, Thomsen,L, Karlsen, V, Boesen,H, Johansen, C. (2007). Quality of life in Danish colorectal cancer patients with and without a stoma. Supportive Care Cancer, 15(5), 505513

Russell S. (2017). Physical activity and exercise after stoma surgery: overcoming the barriers. Br J 
Sechrist, Karen, R., Walker, Susan, N, Pender, Nola, J. (1987). Health Promotion Model -

Instruments to Measure HPM Behavioural Determinants: Exercise Benefits/Barriers Scale [EBBS]

(Adult Version). Retrieved from

http://deepblue.lib.umich.edu/bitstream/handle/2027.42/85354/EBBS-

English_Version.pdf?sequence=2.

Simmons KL, Smith JA, Bobb KA, Liles LL. (2007). Adjustment to colostomy: stoma acceptance, stoma care self- efficacy and interpersonal relationships. J Adv Nurs.60 (6) :627-35.

Taylor, P (2005). An introduction to stomas: Reasons for their formation. Nursing Times, 101 (29), 63.

Vonk-Klassenn SM, de Vocht HM, den Ouden MEM, Eddes EH, Schuurmans MJ. (2016) Ostomyrelated problems and their impact on quality of life of colorectal cancer ostomates: a systematic review. Qual Life Res. 25: 125-133.

World Health Organization (2018). More active people for a healthier world: draft global action plan on physical activity 2018-2030. Physical activity for health, Seventy-first World Health Assembly A71/18. Provisional agenda item 12.2 22. Report by the Director-General, March 2018. Available at: http://apps.who.int/gb/ebwha/pdf_files/WHA71/A71_18-en.pdf

Wu, K, Chauy, P, Twinn, S (2007) Self-efficacy and quality of life among stoma patients in Hong Kong. Cancer Nursing, 30(3), 186-93

Zunft, H, Friebe, D, Seppelt, B, Widhalm, K, Remaut, M, Vaz, D., Kearney, J, Gibney, M (1999). Perceived benefits and barriers to physical activity in a nationally representative sample in the European Union. public health nutrition, 2 (1), 153-160. 
Figure 1: Stoma type and activity level

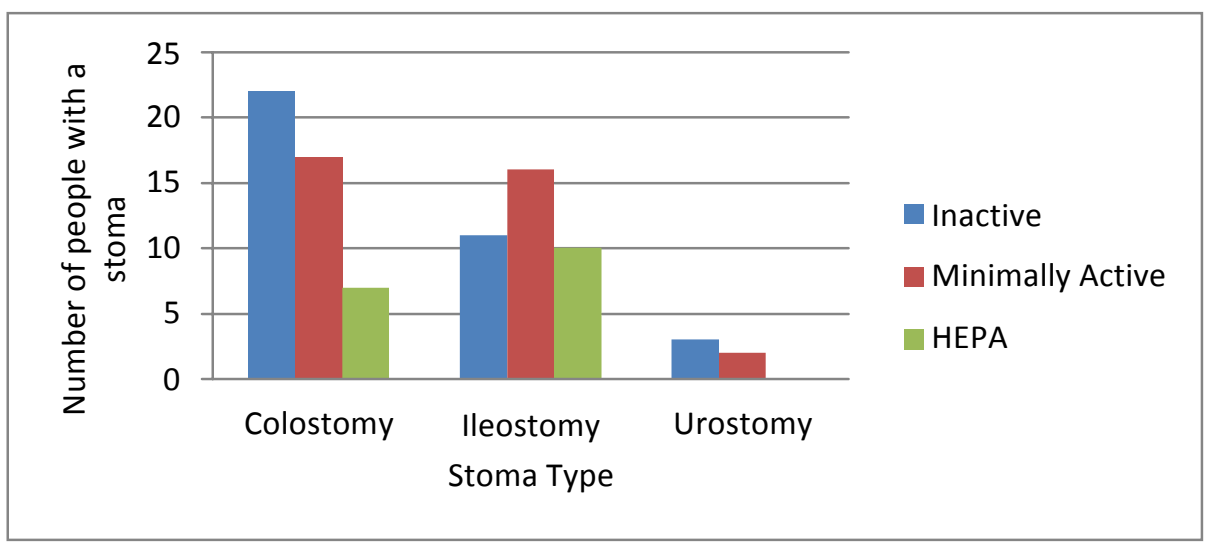


Table 1. Sample Characteristics and MET Intensity Level

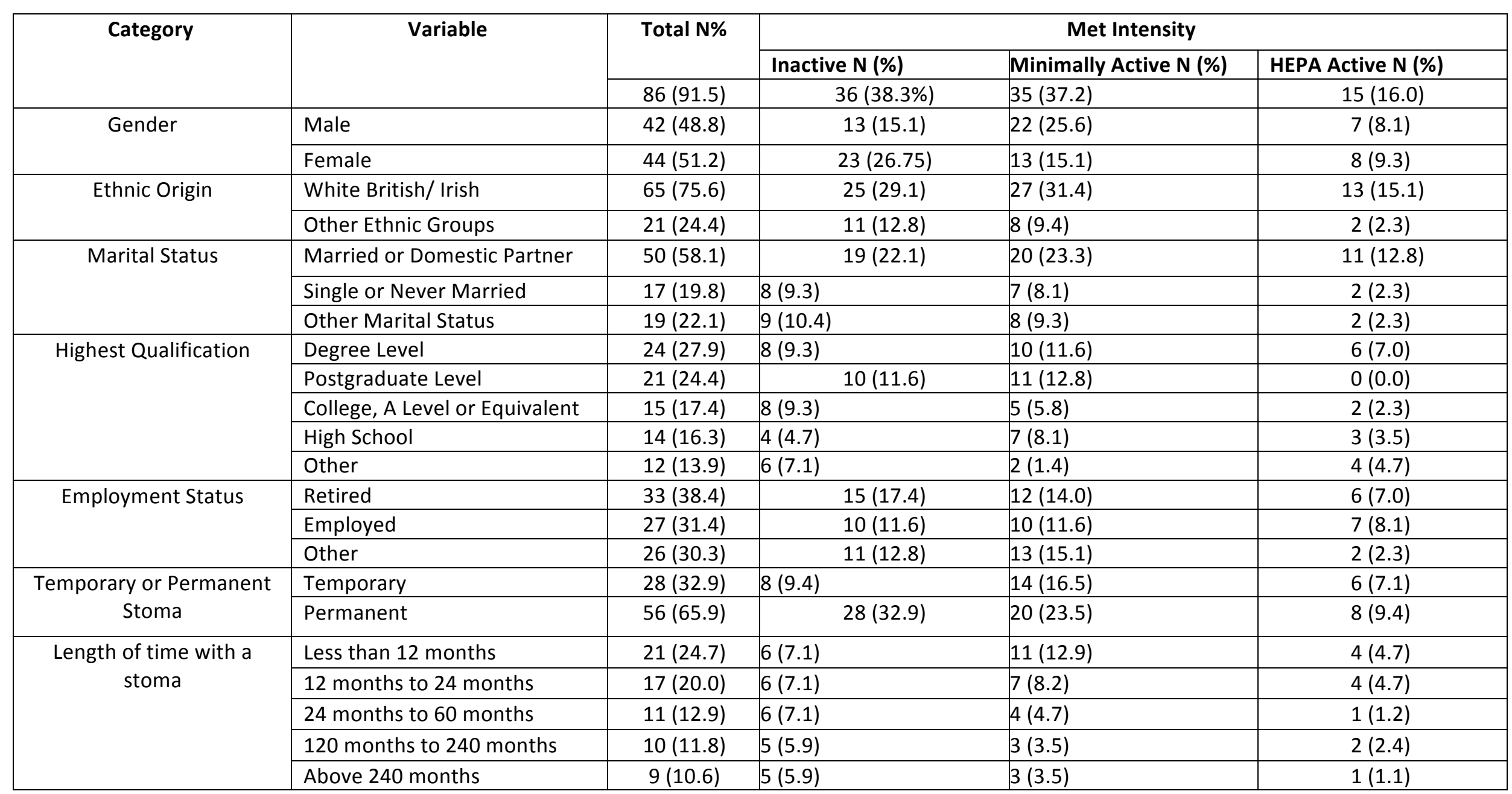

MET: Metabolic Equivalents; HEPA: Health Enhancing Physical Activity 
Table 2. Self-Efficacy for Exercise Scale and MET Intensity Levels

\begin{tabular}{|c|c|c|c|c|c|c|}
\hline Measure & Item & & & \multicolumn{3}{|c|}{ MET Intensity } \\
\hline \multirow{10}{*}{$\begin{array}{l}\text { Self-Efficacy for } \\
\text { Exercise Scale } \\
\text { (SEES) }\end{array}$} & & $\mathrm{N}(\%)$ & Mean $\left(\mathrm{SD}^{+}\right)$ & $\begin{array}{c}\text { Inactive } N \\
\text { (\%) }\end{array}$ & $\begin{array}{c}\text { Minimally } \\
\text { Active N (\%) }\end{array}$ & $\begin{array}{c}\text { HEPA Active } \\
\text { N (\%) }\end{array}$ \\
\hline & You felt pain when exercising & $91(96.8 \%)$ & $2.95(3.02)$ & \multirow{9}{*}{$34(36.2 \%)$} & \multirow{9}{*}{$33(35.1 \%)$} & \multirow{9}{*}{$\begin{array}{l}17(18.1 \%) \\
p=0.001 * *\end{array}$} \\
\hline & You felt depressed & $90(95.7 \%)$ & $3.56(3.09)$ & & & \\
\hline & You felt tired & 91(96.8\%) & $3.79(3.02)$ & & & \\
\hline & You felt stressed & $90(95.7 \%)$ & $4.20(2.99)$ & & & \\
\hline & You did not enjoy it & $90(95.7 \%)$ & $4.81(3.01)$ & & & \\
\hline & You were bored by the programme or activity & $91(96.8 \%)$ & $4.85(4.86)$ & & & \\
\hline & You were too busy with other activities & $92(97.9 \%)$ & $5.19(2.93)$ & & & \\
\hline & You had to exercise alone & $91(96.8 \%)$ & $5.62(3.03)$ & & & \\
\hline & The weather was bothering you & $91(96.8 \%)$ & $5.80(3.06)$ & & & \\
\hline \multicolumn{4}{|c|}{ SEES Total Scale Mean } & $M=30.4$ & $M=46$ & $M=49$ \\
\hline & & EES and MET & tensity Level & \multicolumn{3}{|c|}{$\mathrm{F}=3.04, \mathrm{p}<0.001^{* * *}$} \\
\hline
\end{tabular}

${ }^{+}$Standard Deviation; ${ }^{*} p<=0.05 ;{ }^{* *} p<=0.01 ;{ }^{* * *} p<=0.001{ }^{\text {NS }}=$ Not Significant 
Table 3: Patient Health Questionnaire (PHQ)

\begin{tabular}{|c|c|c|c|c|}
\hline \multirow{2}{*}{ Severity of Depression } & \multicolumn{3}{|c|}{$\begin{array}{c}\text { MET Intensity Level } \\
\text { N (\%) }\end{array}$} & \multirow{2}{*}{ Total Number } \\
\cline { 2 - 5 } & Inactive & Minimally Inactive & HEPA Active & \\
\hline No Depression & $20(33 \%)$ & $27(45 \%)$ & $12(20 \%)$ & 59 \\
\hline Signs of Depression & $19(58 \%)$ & $11(33 \%)$ & $3(9 \%)$ & 33 \\
\hline
\end{tabular}


Table 4. Stoma Related Quality of Life, Social Physique Anxiety, Exercise Benefits and Barriers, and MET Intensity Levels

\begin{tabular}{|c|c|c|c|}
\hline Measure & Item & $\mathrm{N}(\%)$ & Mean $\left(\mathrm{SD}^{+}\right)$ \\
\hline \multirow{7}{*}{ Stoma Related Quality of Life (QoL) } & I become anxious when the pouch is full & $92(97.9 \%)$ & $1.76(0.94)$ \\
\hline & My stoma makes me feel sexually unattractive & $92(97.9 \%$ & $1.49(1.17)$ \\
\hline & I feel embarrassed about my body because of my stoma & $93(98.9 \%)$ & $1.43(1.09)$ \\
\hline & My stoma limits the clothes I can wear & $93(98.9 \%)$ & $1.35(0.96)$ \\
\hline & I worry the pouch will loosen & $92(97.9 \%)$ & $0.95(0.99)$ \\
\hline & QoL Total Scale Mean (SD) & $19.3(12.98)$ & \\
\hline & QoL and MET Intensity Level & $F=0.40, p=0.67^{N S}$ & \\
\hline \multirow{7}{*}{ Social Physique Anxiety Scale (SPAS) } & In the presence of others, I feel apprehensive about my physique & $88(93.6 \%)$ & $2.71(1.30)$ \\
\hline & $\begin{array}{l}\text { It would make me uncomfortable to know others were evaluating my } \\
\text { physique }\end{array}$ & $88(93.6 \%)$ & $2.87(1.27)$ \\
\hline & $\begin{array}{l}\text { Unattractive features of my physique make me nervous in social } \\
\text { situations }\end{array}$ & 87 (92.6\%) & $2.91(1.32)$ \\
\hline & When it comes to displaying my physique to others, I am a shy person & $88(93.6 \%$ & $3.00(1.33)$ \\
\hline & $\begin{array}{l}\text { When in a bathing suit, I often feel nervous about how well } \\
\text { proportioned by body is }\end{array}$ & $85(90.4 \%)$ & $3.08(1.47)$ \\
\hline & SPAS Total Scale Mean (SD) & $30.83(7.9)$ & \\
\hline & SPAS and MET Intensity Level & $F=1.97, p=0.15^{\mathrm{NS}}$ & \\
\hline \multirow{7}{*}{ Perceived Benefits to Exercise (EBBS) } & I will prevent heart attacks by exercising & $91(96.8)$ & $2.20(0.61)$ \\
\hline & Exercise increases my level of physical fitness & $90(95.7)$ & $2.18(0.57)$ \\
\hline & My muscle tone is improved with exercise & $91(96.8)$ & $2.16(0.54)$ \\
\hline & Exercises increases my muscle strength & $91(96.8)$ & $2.15(0.51)$ \\
\hline & Exercise improves the functioning of my cardiovascular system & $91(96.8)$ & $2.14(0.59)$ \\
\hline & $\begin{array}{ll}\text { EBBS (Benefits) Total Scale Mean (SD) } \\
\end{array}$ & $53.5(12.7)$ & \\
\hline & EBBS (Benefits) and MET Intensity Level & $F=6.9, p=0.02^{*}$ & \\
\hline \multirow{7}{*}{ Perceived Barriers to Exercise (EBBS) } & Exercise tires me & $91(96.8)$ & $2.02(0.69)$ \\
\hline & I am fatigued by exercise & $91(96.8)$ & $1.95(0.86)$ \\
\hline & Exercise is hard work for me & $91(96.8)$ & $1.86(0.77)$ \\
\hline & Exercise takes too much of my time & $91(96.8)$ & $1.50(0.96)$ \\
\hline & Places for me to exercise are too far away & $91(96.8)$ & $1.41(0.89)$ \\
\hline & EBBS (Barriers) Total Scale Mean (SD) & $22.7(6.6)$ & \\
\hline & EBBS (Barriers) and MET Intensity Level & $F=3.97, p=0.24^{N}$ & \\
\hline Total EBBS Scale & & & \\
\hline
\end{tabular}


EBBS Total Scale Mean (SD) 74 (15.8)

[N=91]

EBBS Total Scale and MET Intensity Level $F=8.2, p=0.01^{* *}$

${ }^{+}$Standard Deviation; ${ }^{*} \mathrm{p}<=0.05 ;{ }^{*} \mathrm{p}=<0.01,{ }^{\mathrm{NS}}=$ Not Significant 
